\title{
BINARY SELF-DUAL CODES OF LENGTH 24
}

\author{
BY VERA PLESS ${ }^{1}$ AND N. J. A. SLOANE
}

Communicated by Olga Taussky Todd, February 28, 1974

\begin{abstract}
There are 26 distinct indecomposable self-dual codes of length 24 over $G F(2)$, including unique codes of minimum weights 8 and 6 , whose groups are, respectively, the Mathieu group $M_{24}$ and the maximal subgroup of index 1771 in $M_{24}$. For each code we give the order of its group, the number of equivalent codes, and its weight distribution.
\end{abstract}

1. Introduction. An $[n, k]$ code $C$ is a $k$-dimensional subspace of the vector space of all $n$-tuples of 0 's and 1's with mod 2 addition. The dual code $C^{\perp}=\{u: u \cdot v=0$ for all $v \in C\}$ is an $[n, n-k]$ code. $C$ is self-orthogonal if $C \subset C^{\perp}$, self-dual if $C=C^{\perp}$. Self-dual codes exist whenever the length $n$ is even. The weight of a vector is the number of its nonzero components, and the minimum weight of $C$ is the minimum weight of any nonzero codeword. The weight distribution of $C$ is the set $\left\{\alpha_{0}, \alpha_{1}\right.$, $\left.\cdots, \alpha_{n}\right\}$, where $\alpha_{i}$ is the number of codewords of weight $i$.

The group $G(C)$ of a code $C$ is the set of all permutations of the coordinates which send $C$ into itself set-wise. Two codes are equivalent if there is a coordinate permutation sending one into the other. The number of codes equivalent to $C$ is $n ! /$ order of $G(C)$. The direct sum of codes $C^{\prime}$ and $C^{\prime \prime}$, written $C^{\prime} \oplus C^{\prime \prime}$, is $\left\{(u, v): u \in C^{\prime}, v \in C^{\prime \prime}\right\}$. If $C=C^{\prime} \oplus C^{\prime \prime}$, where $C^{\prime}$ and $C^{\prime \prime}$ are nonzero, then $C$ is decomposable. Otherwise $C$ is indecomposable.

Pless [4] classified all self-dual codes of length $\leqslant 20$, Conway (unpublished) found the 9 self-dual codes of length 24 in which the weight of every codeword is a multiple of 4 , and Niemeier [2] found the 24 even unimodular lattices in dimension 24,9 of which correspond to the codes found by Conway.

AMS (MOS) subject classifications (1970). Primary 94A10; Secondary 05A15, 15 A03.

1 The work of the first author was supported in part by Project MAC, an MIT interdepartmental laboratory sponsored by the Advanced Research Projects Agency, Department of Defense, under Office of Naval Research Contract N00014-70-A-0362-0001. 
We have found that there are 8 inequivalent, indecomposable self-dual codes of length 22 , and 26 of length 24 . The latter are shown in Table I, which gives for each code a basis, the order of its group, the number of codes equivalent to it (written as a multiple of $\nu=1 \cdot 3 \cdot 5 \cdot 7 \cdot \ldots \cdot 23=316$, $234,143,225$ ), and the weight distribution $\alpha_{4}, \alpha_{6}, \cdots, \alpha_{12}$ (omitting $\alpha_{0}$ $=1, \alpha_{2}=\alpha_{\text {odd }}=0, \alpha_{i}=\alpha_{24-i}$ for $i>12$ ). Full details of the enumeration will appear in [5].

2. Self-orthogonal codes of minimum weight 4. Table I was obtained by classifying the codes according to minimum weight. A self-dual code of minimum weight 2 is decomposable. For minimum weight 4 we use

THEOREM 1. Let $C$ be an indecomposable self-orthogonal code generated by codewords of weight 4. Then $C$ is one of the codes $d_{n}(n=4$, $6,8, \cdots), e_{7}$, or $E_{8}$, generated by the rows of the following matrices:

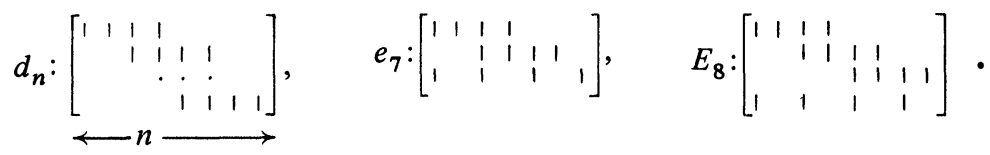

Furthermore a self-dual code containing $E_{8}$ as a subcode is decomposable.

Let $C$ be an indecomposable self-dual code of length 24 , and let $C^{\prime}$ be the subcode generated by codewords of weight 4 . By Theorem $1, C^{\prime}$ has the form $d_{n_{1}} \oplus \cdots \oplus d_{n_{l}} \oplus e_{7} \oplus \cdots \oplus e_{7}$. We considered all such $C^{\prime}$ and all ways of extending $C^{\prime}$ to a self-dual code. For each code we computed the order of its group. In this way all the codes of minimum weight 4 were obtained.

The notation used to specify the basis vectors is best illustrated by an example. The code $J_{24}$ generated by the rows of (1)

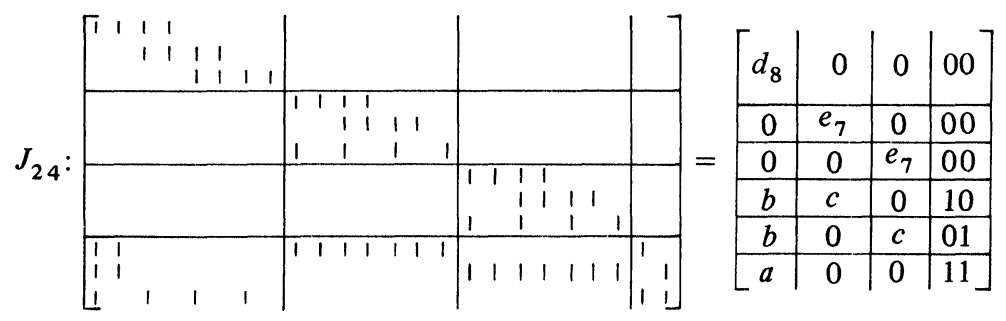

where $a=101010 \ldots 10, b=110000 \ldots 00, c=111 \ldots 1$, is written $d_{8} e_{7}^{2}$ 
$+2 /$ bco $10 /$ boc $01 / a o^{2} 1^{2}$, where the +2 indicates two coordinates which do not meet any codeword of weight 4. $a^{\prime}$ denotes $a+b=011010 \ldots 10$. We omit the full details of $W_{24}, X_{24}, Y_{24}$.

3. Minimum weight 6 and 8. It is known [3], [1] that the [24, 12] Golay code is the unique code of minimum weight 8 , and that its group is the Mathieu group $M_{24}$.

We determined that there is a unique self-dual code of minimum weight 6 , which is generated (in Todd's [6] notation) by the set of 64 nonspecial hexads associated with any set of 6 mutually complementary tetrads in the Golay code. Its group is a maximal subgroup of index 1771 in $M_{24}$.

\section{TABLE I}

Indecomposable Self-Dual Codes of Length 24 (Page 1)

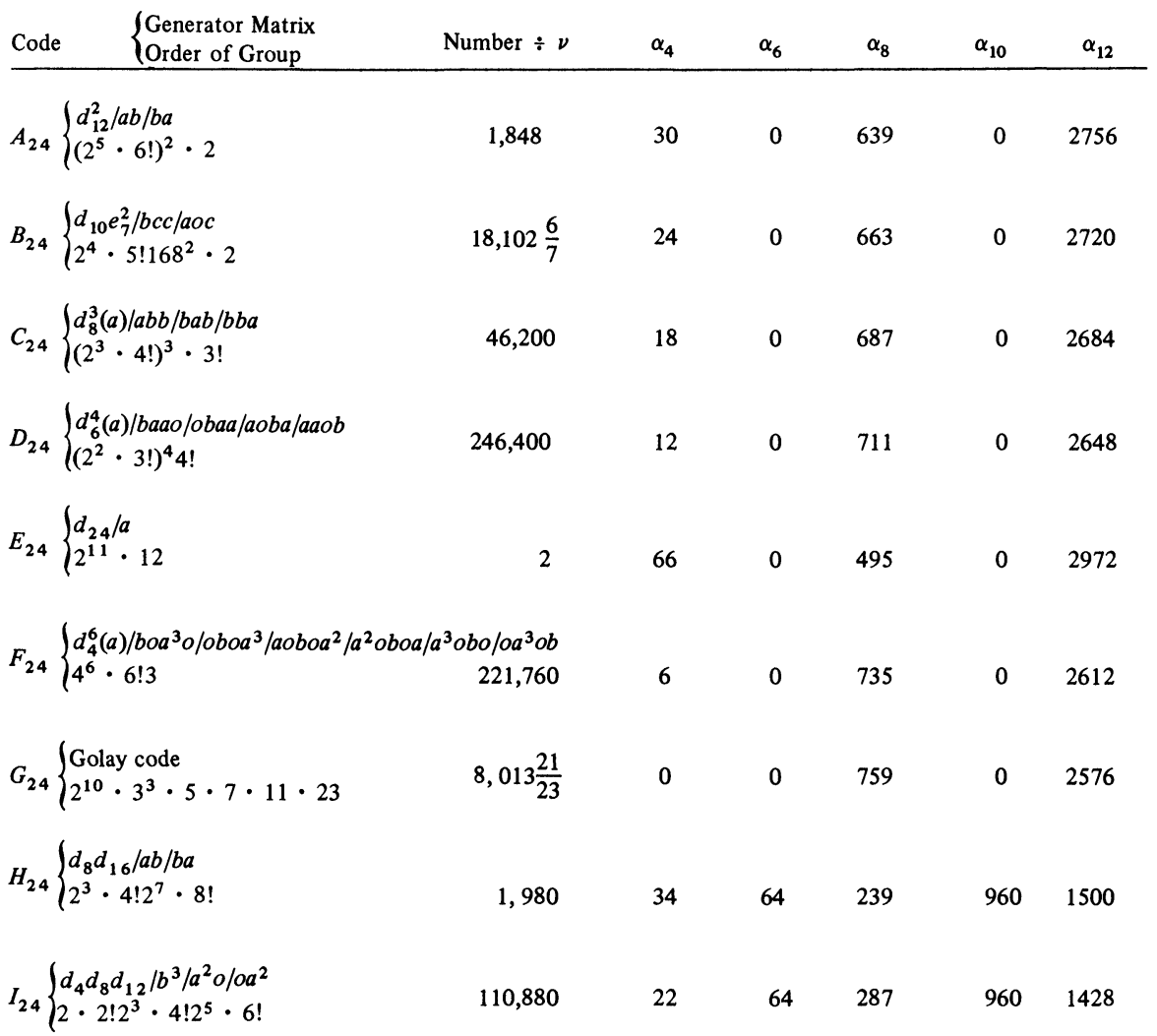


TABLE I

Indecomposable Self-Dual Codes of Length 24 (Page 2)

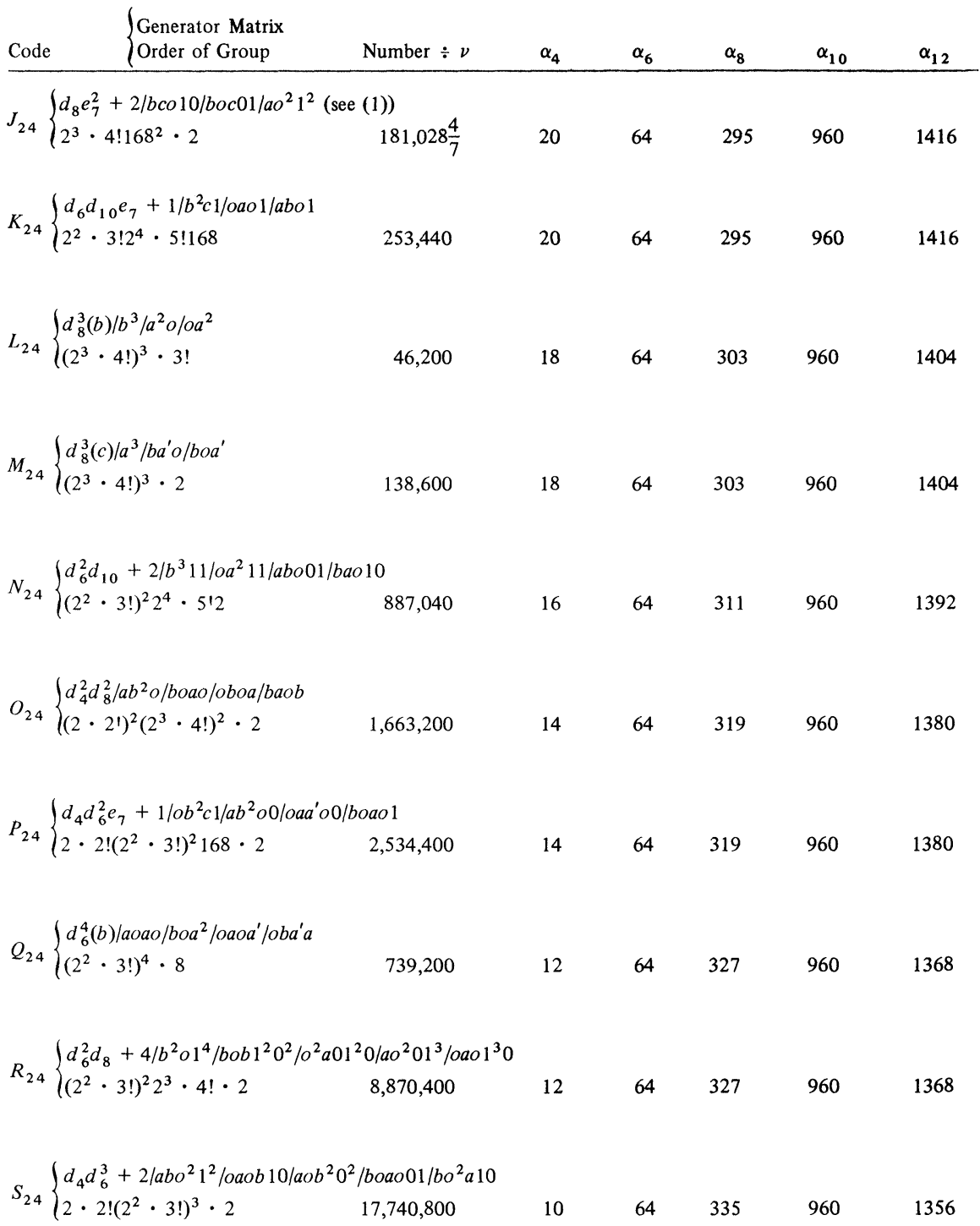

4. General enumeration theorems. The following theorems, and others, were used to check Table I.

THEOREM 2. Let $\alpha_{C}(x)=\sum_{i=0}^{n} \alpha_{i} x^{i}$ be the weight enumerator of $C$. Then 


$$
\sum \alpha_{C}(x)=\prod_{j=1}^{n / 2-2}\left(2^{j}+1\right) \cdot\left[2^{n / 2-1}\left(1+x^{n}\right)+\sum_{2 \mid i}\left(\begin{array}{l}
n \\
i
\end{array}\right) x^{i}\right],
$$

where the sum extends over all self-dual codes $C$ of even length $n$.

THEOREM 3. If $n$ is even, the number of self-dual codes with length $n$ and minimum weight $\geqslant 4$ is

$$
\begin{aligned}
& \sum_{i=0}^{n / 2} \frac{(-1)^{i} n !}{2^{i} i !(n-2 i) !} \prod_{j=1}^{n / 2-i-1}\left(2^{j}+1\right) \\
& \text { TABLE I } \\
& \text { Indecomposable Self Dual Codes of Length } 24 \text { (Page 3) } \\
& \text { Code } \quad\left\{\begin{array}{l}
\text { Generator Matrix } \\
\text { Order of Group }
\end{array}\right. \\
& T_{24}\left\{\begin{array}{l}
d_{4}^{4} d_{8} / b a b a b / b a^{2} o a / o a b^{2} a^{\prime} / a o b a^{2} / b^{2} o a a^{\prime} \\
4^{4} \cdot 2^{3} \cdot 4 ! \cdot 8
\end{array}\right. \\
& \begin{array}{lllll}
4,989,600 & 10 & 64 & 335 & 960
\end{array} \\
& 1356
\end{aligned}
$$

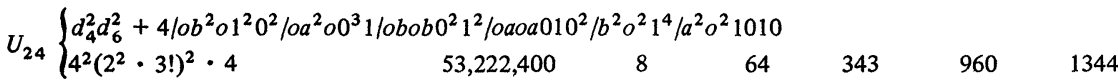

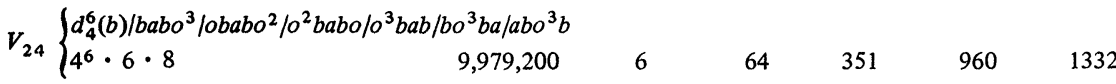

$$
\begin{aligned}
& W_{24}\left\{\begin{array}{l}
d_{4}^{3} d_{6}+6 / \cdots \\
4^{3} \cdot 2^{2} \cdot 3 ! \cdot 3 ! \cdot 2
\end{array} \quad 106,444,800 \quad 6 \quad 64 \quad 351 \quad 960 \quad 1332\right. \\
& X_{24}\left\{\begin{array}{l}
d_{4}^{4}+8 / \cdots \\
4^{4} \cdot 4 ! \cdot 2
\end{array}\right. \\
& \begin{array}{llllll}
159,667,200 & 4 & 64 & 359 & 960 & 1320
\end{array} \\
& Y_{24}\left\{\begin{array}{l}
d_{4}^{2}+16-o / \cdots \\
2^{11} \cdot 3^{2}
\end{array}\right. \\
& \begin{array}{llllll}
106,444,800 & 2 & 64 & 367 & 960 & 1308
\end{array} \\
& Z_{24}\left\{\begin{array}{l}
\text { see } \$ 3 \\
2^{10} \cdot 3^{3} \cdot 5
\end{array}\right. \\
& \begin{array}{llllll}
14,192,640 & 0 & 64 & 375 & 960 & 1296
\end{array} \\
& 556,041,557 \frac{86}{1127} \cdot \nu
\end{aligned}
$$

\section{REFERENCES}

1. E. F. Assmus, Jr. and H. F. Mattson, Perfect codes and the Mathieu groups, Arch. Math. (Basil) 17 (1966), 121-135. MR 34 \#4050.

2. H.-V. Niemeier, Definite quadratische Formen der Dimension 24 und Diskriminante 1, J. Number Theory 5 (1973), 142-178. 
3. Vera Pless, On the uniqueness of the Golay codes, J. Combinatorial Theory 5 (1968), 215-228.

4. - A classification of self-orthogonal codes over $G F(2)$, Discrete Math. 3 (1972), 209-246. MR $46 \# 3200$.

5. Vera Pless and N. J. A. Sloane, On the classification and enumeration of selfdual codes J. Combinatorial Theory.

6. J. A. Todd, A representation of the Mathieu group $M_{24}$ as a collineation group, Ann. Mat. Pura Appl. (4) 71 (1966), 199-238. MR 34 \#271 3,

ELECTRICAL ENGINEERING DEPARTMENT, MASSACHUSETTS INSTITUTE OF TECHNOLOGY, CAMBRIDGE, MASSACHUSETTS 02139

BELL TELEPHONE LABORATORIES INC., MURRAY HILL, NEW JERSEY 07974 